\title{
ILC2s: New Actors in Tumor Immunity
}

\author{
Giuseppe Ercolano, Maryline Falquet ${ }^{\dagger}$, Giulia Vanoni ${ }^{\dagger}$, Sara Trabanelli and \\ Camilla Jandus*
}

Department of Oncology, Ludwig Institute for Cancer Research Lausanne, Centre Hospitalier Universitaire Vaudois (CHUV), University of Lausanne, Lausanne, Switzerland

Innate lymphoid cells (ILCS) represent the most recently identified family of innate lymphocytes that act as first responders, maintaining tissue homeostasis and protecting epithelial barriers. In the last few years, group 2 ILCs (ILC2s) have emerged as key regulators in several immunological processes such as asthma and allergy. Whilst ILC2s are currently being evaluated as novel targets for immunotherapy in these diseases, their involvement in tumor immunity has only recently begun to be deciphered. Here, we provide a comprehensive overview of the pleiotropic roles of ILC2s in different tumor settings. Furthermore, we discuss how different therapeutic approaches targeting ILC2s could improve the efficacy of current tumor immunotherapies.

OPEN ACCESS

Edited by:

Nicolas Jacquelot,

Walter and Eliza Hall Institute of Medical Research, Australia

Reviewed by: Omid Akbari,

University of Southern California,

United States

Brian Kim,

Washington University School of Medicine in St. Louis, United States

*Correspondence:

Camilla Jandus camilla.jandus@gmail.com

tThese authors have contributed equally to this work

Specialty section:

This article was submitted to

NK and Innate Lymphoid Cell Biology, a section of the journal

Frontiers in Immunology

Received: 01 October 2019 Accepted: 14 November 2019 Published: 03 December 2019

Citation:

Ercolano G, Falquet M, Vanoni G, Trabanelli S and Jandus C (2019) ILC2s: New Actors in Tumor Immunity. Front. Immunol. 10:2801. do: 10.3389/fimmu.2019.02801
Keywords: patients, immunotherapy, cancer, ILC2, IL-33, ST2

\section{INTRODUCTION}

ILCs are the most recently described family of innate immune cells that play a key role in the preservation of epithelial integrity and tissue immunity (1). ILCs are rapidly activated by both tissue and immune cell-derived signals providing the first line of defense against bacterial, viral and helminthic infections (2-6). However, ILCs need to be tightly regulated, given that their uncontrolled activation and proliferation has been shown to contribute to severe inflammation and damage in gut, lung, skin, and liver (7). ILCs are classified into three different groups, according to the expression of specific transcription factors and surface markers, and based on their cytokine secretion profile (8).

In humans, ILC1s define the T-bet-dependent ILC subset that mainly produce IFN $\gamma$ and TNF $\alpha$ (9). ILC3s rely on ROR $\gamma \mathrm{T}$ for their development and express CD117 (also referred to as c-Kit) on their cell surface (10). ILC2s comprise the GATA-3-dependent ILC subset that is also characterized by the expression of the prostaglandin D2 receptor 2 (CRTH2), the IL-33 receptor (IL1RL1 also referred as ST2) and by variable levels of c-Kit (11). More recently, Nagasawa and colleagues showed that the killer cell lectin-like receptor subfamily G member 1 (KLRG1) is a surface marker that arises during ILC2 development in humans (12). KLRG1 is a co-inhibitory receptor already reported to be expressed also by $\mathrm{CD} 4^{+}$and $\mathrm{CD} 8^{+} \mathrm{T}$ cells as well as by NK cells, that binds to the members of the cadherin family $(13,14)$. In mice, the ILC2 phenotype is characterized by the surface expression of both ST2 and KLRG1 (15). Notably, ST2 ${ }^{+} \mathrm{KLRG1}^{+/-}$ILC2s are defined as natural ILC2s (nILC2s)

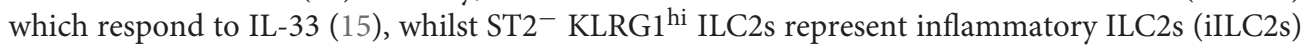
reported to differentiate during infections. iILC2s are highly responsive to IL-25, but not to IL-33, and are able to differentiate into ILC3-like cells under type-17 stimulation, thus defining a distinct subset from nILC2s. ILC2s are able to respond to a wide range of soluble mediators like alarmins [IL-25, IL-33, and thymic stromal lymphopoietin (TSLP)], survival cytokines (such as IL-2, IL-9, and IL-7) and eicosanoids. In addition, ILC2s have been shown also to respond to neuropeptides, including neuromedin $\mathrm{U}(\mathrm{NMU})$, vasoactive intestinal peptide (VIP), and calcitonin gene-related peptide (CGRP) $(3,16-20)$. More precisely, it has been shown that neuropeptides released by 
pulmonary endocrine cells (PNECs) can stimulate resident ILC2s to produce cytokines, such as IL-5, which in turn support downstream type-2 immune responses (21). Similarly, VIP can stimulate IL-5 release by ILC2s, regulating eosinophil homeostasis in intestinal tissues (22). On the contrary, an opposite role for the CGRP was described, as it can negatively modulate ILC2 effector functions (i.e., cytokine production) in the context of lung inflammation and also during helminth infections $(23,24)$. It has also been reported that ILC2s in the small intestine, express high levels of the $\beta_{2}$ - adrenergic receptor $\left(\beta_{2}-\mathrm{AR}\right)$, which acts as a negative regulator of the ILC2-mediated anti-inflammatory response (25).

Once activated, ILC2s secrete type 2 cytokines, such as IL-4, IL-5, IL-9, IL-13, and amphiregulin (AREG), that are involved in airway responses, helminth expulsion, and tissue repair (26). More recently, it has been reported that activated ILC2s are able to produce prostaglandin D2 (PGD2) that acts in an autologous manner supporting ILC2 function via the CRTH2 receptor (27). A detrimental role of ILC2s in chronic inflammation is suggested by their increased frequency in the peripheral blood of asthma and chronic rhinosinusitis patients; and additionally, the secretion of AREG by intrahepatic ILC2s is thought to contribute to the process of fibrogenesis in liver diseases $(28,29)$.

However, in cancer, the role of ILC2s is still controversial. Elevated numbers of ILC2s have been found in many IL-33enriched tumors, such as breast, gastric and prostate cancer $(30-32)$ as IL-33 is an ILC2 activator that can promote tumor growth, metastatic dissemination and angiogenesis (33). The ILC2 pro-tumorigenic activity is mainly ascribed to the IL-33triggered IL-4 and IL-13 production. These cytokines have been reported to support tumor development and progression (34), in part by the recruitment and activation of monocytic myeloidderived suppressor cells (M-MDSCs) that are considered potent inhibitors of the anti-cancer immune response (35). In addition, AREG produced by ILC2s, can further suppress the anti-tumor immune response by boosting the activity of regulatory $\mathrm{T}$ cells (Tregs) (36). Conversely, ILC2-produced IL-5 promotes blood and tissue eosinophilia that correlates with reduced tumorigenicity and tumor progression in mice (37). In this review, we summarize the current knowledge concerning the presence and functional characteristics of ILC2 populations in different tumors, using both patient samples and murine tumor models (Figure 1). Furthermore, we discuss potential strategies to exploit ILC2 biology to improve the efficacy of current tumor immunotherapies.

\section{ILC2s IN HEMATOLOGICAL MALIGNANCIES}

Hematological malignancies represent the fourth most common type of cancer (38). ILCs are a rare cell population, representing $\sim 0.4 \%$ of total circulating peripheral blood lymphocytes in humans (39), however, we have reported that ILC2s are expanded in the peripheral blood of acute promyelocytic leukemia (APL) patients at diagnosis, compared to healthy donors. In particular, we found that ILC2s have a central role in the establishment of an immunosuppressive axis, dictated by the tumor-derived factors PGD2 and B7H6 and their ILC2 receptors CRTH2 and NKp30, respectively. This interaction triggers the production of IL-13 which in turn recruits M-MDSCs supporting the growth of cancer cells [(31); Figure 1, left lower corner]. These findings were also confirmed in an APL mouse model raising the possibility of finding the same axis in other tumors, including solid tumors such as prostate cancer (see "ILC2s in prostate cancer" section).

In contrast, in treatment-naïve patients with acute myeloid leukemia (AML), we and others have observed an expansion of ILC1s. There was no detection of a change in ILC2 frequency but we observed a lower production of IL-5 and IL-13 following in vitro short-term activation with phorbol 12-myristate 13acetate (PMA) plus ionomycin (40). In this context, the increased ILC1 frequency might be due to the conversion of ILC3s and/or ILC2s into ILC1s driven by tumor-derived factors, among others TGF $\beta$. A putative anti-tumor role of ILC2s has been proposed in a subcutaneous lymphoma mouse model, where sustained production of IL-33 induced the upregulation of CXCR2 on EL4 thymoma cells, the expansion of ILC2s and the concomitant production of CXCR2 ligands (CXCLs). These ligands, mainly CXCL1 and CXCL2, induced apoptosis in a limited proportion of lymphoma cells, thus limiting tumor progression [(41); Figure 1, right middle panel].

\section{ILC2S IN UROGENITAL TRACT CANCERS}

\section{ILC2s in Prostate Cancer}

Prostate cancer is the most common non-cutaneous malignancy in men and responsible for about 20\% of male cancerrelated deaths (42). Despite the different therapeutic approaches, including the use of immune checkpoint inhibitors, limited clinical benefits have been observed in patients (43). In this context, the tumor microenvironment (TME) seems to play a key role in driving prostate cancer progression and chemoresistance $(44,45)$. Focusing on ILCs in prostate cancer patients, we have shown that ILC2 levels positively correlate with tumor stage and with M-MDSC frequency (31). Additionally, DU145 and PC3 prostate cancer cells secrete the ILC2 activator PGD2 and express high levels of B7H6, the ligand of NKp30, corresponding with the immunosuppressive axis found in APL patients. Using the spontaneous TRAMP model, in which mice develop orthotopic prostate tumors from puberty (46), we observed an increase of ILC2s both in the blood and the tumor supporting our findings in prostate cancer patients (31). Conversely, Saranchova et al. have showed that ILC2s can acquire anti-tumor activities by influencing the effector functions of cytotoxic lymphocytes, through the release of IL-5 and IL-13 acting on DCs. They used the pTAP-1-EGFP-stably-transfected LMD cell line, derived from a metastatic prostate cancer mouse model, in which TAP1 activation in tumor cells indirectly correlates with MHC-I and EGFP expression. In order to mimic metastatic prostate cancer conditions in vivo, the authors isolated ILC2s from tumors of donor mice and cultured them with the LMD cell lines, $\mathrm{CD}^{+}$dendritic cells (DCs), ovalbumin (OVA) peptide as well as $\mathrm{CD}^{+}$OT-1 T cells. They observed an increased 


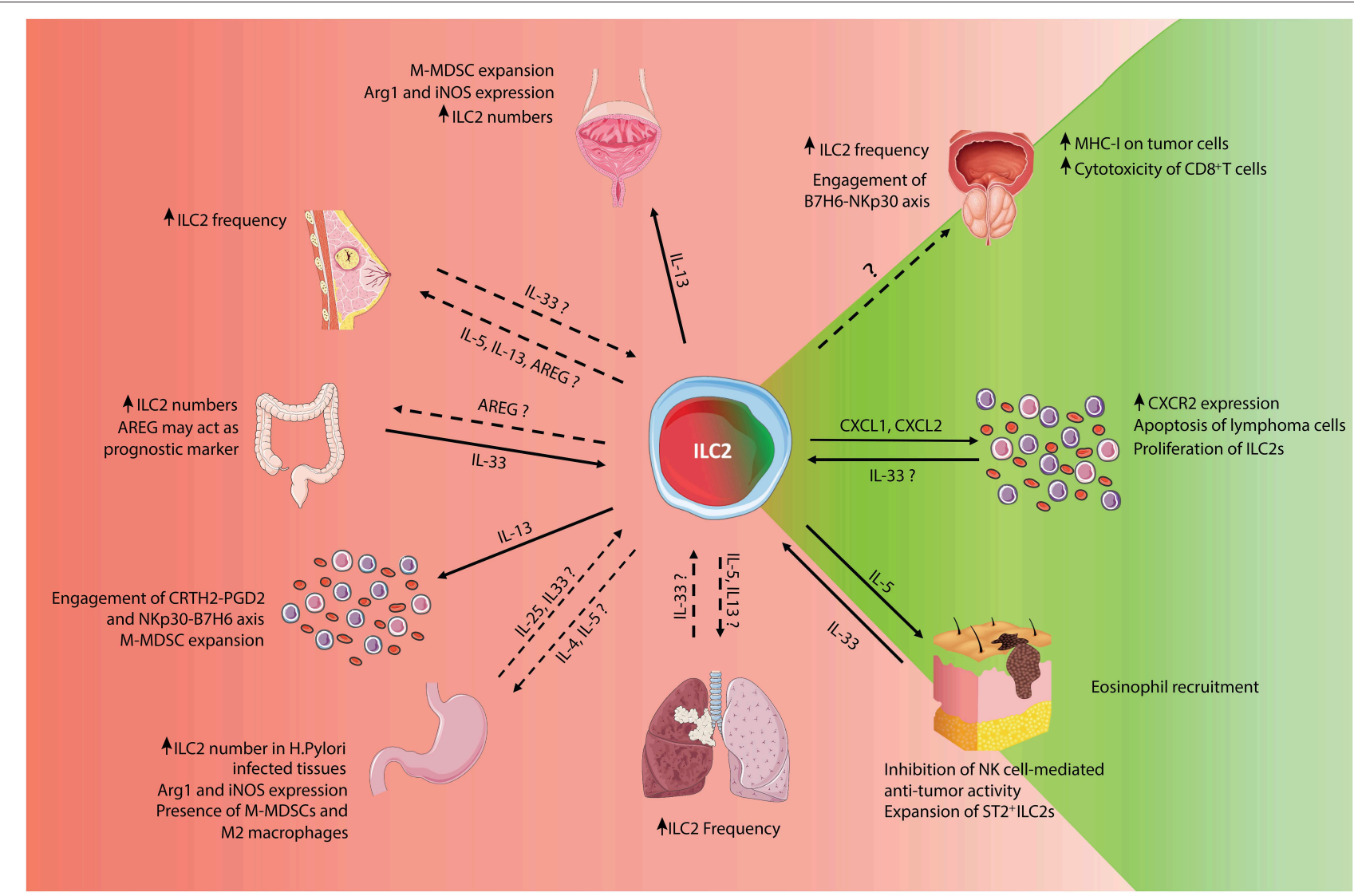

FIGURE 1 | Schematic representation of pro- and anti-tumor roles of ILC2s. Summary of the known pro- and anti-tumor roles of ILC2s, classified by tumor types. For bibliographic details refer to the work cited in the main manuscript.

expression of EGFP, indicating that the LMD cells had been stimulated to express MHC-I on their surface by ILC2s. This suggests that ILC2s via direct interaction or cytokine secretion, facilitate antigen presentation and the recognition of tumor cells by $\mathrm{T}$ cells, thus improving the anti-tumor adaptive response [(47); Figure 1, right upper panel]. The use of tumor-bearing ILC2 $\mathrm{KO}$ mice transferred with ILC2s isolated from wild-type donor mice might represent a good strategy to dissect the contribution of ILC2s in the T-cell mediated antitumor responses.

\section{ILC2s in Bladder Cancer}

Bladder cancer (BC) is broadly divided into two major stages: non-muscle-invasive (NMIBC) and muscle-invasive bladder cancer (MIBC) and is the ninth most common cancer worldwide (48). NMIBC standard treatment involves intravesical instillation of the Bacillus Calmette-Guérin antigen (BCG) (49), whereas MIBC treatment involves neoadjuvant cisplatinbased chemotherapy (NAC) followed by radical cystectomy (50). Despite these approaches, the rate of recurrence of $\mathrm{BC}$ remains high (51). To better understand the reasons behind BC treatment failure, immune cell distribution was analyzed in the urine of NMIBC patients during BCG treatment and ILC2s were found to be the most abundant innate lymphoid cell subpopulation present (52). ILC2 frequency positively correlates with M-MDSC frequency but not with $\mathrm{T}$ cell numbers suggesting that ILC2s may promote the expansion of M-MDSCs. This correlation has also been confirmed in the blood of patients with MIBC. In addition, ILC2-associated cytokines measured in blood and urine samples of NMIBC and MBIC patients showed a significantly elevated level of IL-13 compared to healthy donors. IL-13 secretion could explain the ILC2-dependent recruitment of MMDCS which were shown to express the IL-13 receptor $\alpha 1$ (IL-13R $\alpha 1$ ). At mRNA level, the immunosuppressive properties of IL-13 were demonstrated with upregulation of monocytic suppressive markers such as arginase $1(\operatorname{Arg} 1)$, inducible nitric oxide synthase (iNOS) and $\mathrm{C} / \mathrm{EBP} \beta$ [(52); Figure 1, upper central panel]. These results highlight the concept that the $\mathrm{BC}$ immunosuppressive environment is, at least in part, driven by ILC2-derived IL-13 that may be contributing to the failure of current BC therapies. Furthermore, the ratio between $\mathrm{T}$ cells and M-MDSCs may also have an impact on the response to treatment, since patients with a high $\mathrm{T}$ cell/MDSC ratio show improved survival with reduced risk of recurrence. However, more research is needed to better understand the role of ILC2s in this type of cancer. 


\section{ILC2s IN CANCERS OF THE GASTROINTESTINAL SYSTEM}

\section{ILC2s in Colorectal Cancer}

Colorectal cancer (CRC) is the third and second most common cancer diagnosed in men and women, respectively (53). CRC mortality rate has decreased over recent years due to improved cancer screening methods (54). A variety of genetic, environmental and nutritional factors play a key role in the pathogenesis and progression of CRC (55). Several immune cell populations infiltrate the CRC TME by modulating the tumor response (56). Among them, ILC2s, that are abundant in the intestinal mucosa (57), have been reported in CRC patients, to be recruited to the tumor site suggesting their potential role in CRC development and progression (58). However, there is still no robust data in human or mouse models, clarifying the role of ILC2s in colorectal tumorigenesis. Nevertheless, analysis of human resected CRC specimens has shown that SW480 and SW620 cells at different stages of the disease are positive for IL-33 and its receptor ST2 (59-61). IL-33 has been shown to promote the in vitro proliferation of freshly isolated primary CRC cells (the HT-29 CRC cell line and the murine MC38 cell line), through the activation of the ST2 receptor. The IL-33/ST2 axis activates NF-kB signaling which in turn induces cyclooxygenase2 (COX2) expression and prostaglandin E2 ( $\left.\mathrm{PGE}_{2}\right)$ synthesis, triggering CRC cell proliferation (62). Further evidence for involvement of the IL-33/ST2 axis in CRC pathogenesis comes from an inflammation-driven model in which ST2 deficiency in mice conferred protection against tumor development (61) and secondly from a polyposis mouse model $\left(\mathrm{Apc}^{\mathrm{Min} /+}\right)$, where abrogation of IL-33 signaling reduced the tumor burden, Th2associated cytokine production and mast cell activation (59). Conversely, Akimoto et al. have reported that sST2, a soluble form of the IL-33 receptor, is down-regulated in patient serum and correlates inversely with disease progression. This data has also been confirmed in nude mice, in which injection of short hairpin RNA (shRNA) targeting sST2, triggered tumor development, and progression (60). These findings underline the potential dual role of the IL-33/ST2 axis in colon cancer (63) and the need for further analysis of this pathway in different CRC models. AREG is another important molecule that regulates cancer cell proliferation, invasion and angiogenesis (64) and has been proposed as a prognostic marker in CRC (65). AREG upregulation is associated with increased migration and invasion of CRC cells which is essential for metastasis [(66, 67); Figure 1, left middle panel]. AREG can be produced by different immune cell types under pro-inflammatory conditions, such as mast cells, basophils, tissue resident CD4 T cells (68). However, no data is available to date on ILC2-derived AREG in CRC development and progression.

\section{ILC2s in Gastric Cancer}

With a $65 \%$ overall survival rate, gastric cancer is one of the most common malignancies affecting the digestive system, with more than one million people newly diagnosed each year worldwide (69). However, due to poor population strategies for primary prevention and lack of early symptoms, most patients are diagnosed at an advanced stage with limited benefit from existing therapies (70). The use of immunotherapy for the treatment of metastatic gastric cancer such as pembrolizumab has showed promising effects in Phase I clinical trials (71), but other strategies are still needed to improve patient survival. Gastric tumors are multifactorial in etiology and one of the main risk factors for disease is chronic infection with Helicobacter Pylori $(H$. Pylori) (72). H. Pylori infection causes chronic inflammation of gastric tissue, favoring the development of gastric carcinoma (73). Higher numbers of ILC2s have been observed in the tumors of gastric cancer patients infected with $H$. Pylori, suggesting a role for ILC2s in this immunosuppressive type 2 environment [(74); Figure 1, left lower panel]. Moreover, the frequency of ILC2s in the peripheral blood mononuclear cell (PBMC) compartment is higher in gastric cancer patients than in healthy volunteers and ILC2-associated cytokines, such as IL-4, IL-5, and IL-13, are increased in gastric cancer patients, both at mRNA and protein level in PBMCs and plasma, respectively. In addition, Arg1 and iNOS, expressed in M-MDSCs and M2 macrophages as well as in group 2 ILCs $(75,76)$ were found to be highly expressed at mRNA level in PBMCs of gastric cancer patients (77). Moreover, type 2 cytokines derived from ILC2s have been reported to mediate Arg1 and iNOS secretion by MDSCs and M2 macrophages suggesting a role for ILC2s in promoting M-MDSCs and M2 macrophage phenotype and favoring their immunosuppressive function $(78,79)$. However, using the $\mathrm{gp} 130^{\mathrm{FF}}$ mouse model, validated as a model of spontaneous gastric cancer, Eissmann et al. (80), demonstrate that mast cells, rather than ILC2s, promote tumor growth upon IL-33 stimulation. The authors show that mast cells are more abundant than ILC2s in gastric tumors and secrete macrophagechemoattractant colony-stimulating factor 2 (CSF2), CCL3, and IL-6 in response to activation by tumor-derived IL-33. In ST2 deficient animals (gp $130^{\mathrm{FF}} \mathrm{ST} 2^{-/-}$mice), the authors observed lower tumor burden, which was increased upon adoptive transfer of ST2 ${ }^{+}$wild type bone marrow-derived mast cells (BMMC). Therefore, additional studies with adoptive transfer of ST2 ${ }^{+}$wild type ILC2s could help to determine the individual contribution of mast cells and ILC2s in this cancer setting.

\section{ILC2s IN BREAST CANCER}

Breast cancer is the most common cancer affecting women and its incidence rate in younger women is expected to increase (81). Despite the progress in breast cancer detection and treatment (82), aggressive tumors, such as triple negative breast cancer (TNBC), still lack targeted therapies (83). Immunotherapeutic strategies provide hope of finding new treatment approaches (84), but due to the high heterogeneity of breast cancer (85), much more needs to be done to fully understand the interactions between immune and breast cancer cells (86). ILC2 frequency has been shown to be higher in malignant compared to benign breast tissue in humans (32). Using the 4T1 mammary carcinoma model, Jovanovic et al. have reported an increase in endogenous levels of IL-33, that correlated with cancer progression and metastasis. Using the parental 4T1 cell line overexpressing IL-33, they showed elevated frequencies of IL-5 and IL-13-expressing ILCs in tumor-bearing mice [(33); Figure 1, left upper panel]. 
More precisely, in this model they found that ILC2s trigger tumor progression and metastasis development in response to IL33, sustaining the immunosuppressive milieu that characterizes breast cancer patients. This data suggests that ILC2s could be activated by IL-33 to secrete IL-5 and IL-13 in the 4T1 model of breast cancer, but further investigation is required to confirm this finding also in patients. Moreover, it has been shown that AREG regulates the proliferation and the migration of different mouse and human estrogen-receptor positive $\left(\mathrm{ER} 2^{+}\right)$breast cancer cell lines (87). However, it is still unknown whether ILC2s and ILC2derived AREG are involved in this pro-tumoral axis. The use of ILC2 KO mice could represent a strategy to address the role of AREG-producing ILC2s in the context of breast cancer.

\section{ILC2s IN MELANOMA}

Melanoma is the most aggressive form of skin cancer with a high mortality rate (88). Whilst early stage melanoma is usually curable with surgery, metastatic melanoma is difficult to treat and often fatal. Nevertheless, in the last few years, treatment for metastatic melanoma has advanced due to the introduction of cytotoxic T-lymphocyte-associated antigen-4 (CTLA-4) and the programmed cell-death protein 1 (PD-1) checkpoint inhibitors (89). However, despite these promising discoveries, a high percentage of patients still experience treatment resistance (90) emphasizing the need to find new therapeutic approaches. The TME has been identified recently as a potential target for metastatic melanoma immunotherapy (91). Among the different TME mediators, IL-33 has been reported to inhibit tumor growth in a melanoma mouse model, by stimulating the antitumor activity of $\mathrm{CD}^{+} \mathrm{T}$ cells and natural killer (NK) cells (92). However, this cytokine has also been shown to bind to and expand $\mathrm{ST}_{2}{ }^{+}$tumor-infiltrating ILC2s, characterized by the expression of the immunosuppressive ectoenzyme CD73. In this setting, ILC2s partially antagonized the IL-33 dependent, NK cell-mediated anti-tumor response, as evidenced by cell depleting experiments in which the lack of ILC2 $\mathrm{CD}^{+} 3^{+}$cells led to enhanced NK cell activity and better tumor control (92). This data shows that IL-33 has both a beneficial anti-tumoral role via adaptive immune cells but also a pro-tumoral role via ILC2s. IL-33 is also able to stimulate ILC2s to produce IL-5, a potent eosinophil chemoattractant. Ikutani et al. showed that, in a murine model of metastatic melanoma, the main source of IL-5 was a CD3 ${ }^{\text {neg }}$ population, characterized by the expression of CD90, CD127, CD25, and ST2 (bona fide ILC2s). IL-5 was crucial to induce tumor rejection via eosinophil recruitment, also resulting in reduced lung metastases [(93); Figure 1, right lower panel]. The use of neutralizing antibodies directed against IL-5 may be useful to confirm the involvement of ILC2s in metastatic melanoma.

\section{ILC2s IN LUNG CANCER}

Lung cancer is generally divided into two types, small cell lung cancer (SCLC) and non-small cell lung cancer (NSCLC) (94). It is strongly correlated with cigarette smoking $(95,96)$ and is the most common cause of cancer-related deaths. Different targeted immunotherapies are now being used in lung cancer patients including anti-PD-1 antibodies that have been recently approved for the treatment of SCLC (97-99). Nonetheless, a significant percentage of patients do not respond or develop resistance to treatment, leading to consequent cancer progression $(96,100,101)$. ILC2s constitute the most prominent ILC subset in the respiratory tract under physiologic conditions, although their overall numbers are low (26). They respond rapidly to tissuederived alarmins (102), therefore, unsurprisingly, circulating ILC2s and M-MDSCs were found to be increased in a cohort of 36 lung cancer patients at diagnosis and correlated with a strong type 2 phenotype (103). The expansion of ILC2s in the periphery was accompanied by higher levels of IL-5, IL13, IL-33, and Arg1 in the plasma of lung cancer patients compared to healthy donors. Simoni et al. have also detected ILC2s within lung tumor tissues. However, no functional assays were performed in these studies to define the pro- or antitumor roles of ILC2s in lung cancer [(58); Figure 1, central lower panel]. It can be speculated that the observed strong type 2 phenotype may represent a targetable axis for the development of new immunotherapeutic strategies, fostering the anti-tumor immune response. In contrast to these observations, Carrega et al., reported a reduced frequency of ILC2s in tumors compared to normal lung tissue (104). However, in the absence of sufficient data on the function of ILC2s in lung cancer, it is too early to define their role in this setting.

\section{FUTURE PERSPECTIVES AND CONCLUDING REMARKS}

Tumorigenesis is the result of multiple cell intrinsic (e.g., uncontrolled proliferation, cell migration) and cell extrinsic (e.g., pro-inflammatory or immunosuppressive microenvironment, growth factors, angiogenesis) factors (105). Among the latter, the contribution of the immune system to tumor development and/or tumor cell clearance has become more and more accepted/relevant (106). Even though the impact of ILC2s in malignancy is not currently well defined, the number of studies focusing on the role of ILC2s in tumor immunity has multiplied (107), highlighting the importance of this cell type during cancer development and progression. However, many aspects still need to be elucidated to achieve a better understanding of the mechanisms behind ILC2 pro- and anti-tumoral functions. Moreover, it is known that ILC2s are highly plastic cells that can easily adapt to the environment to which they are exposed (12). Hence, the cytokines present in the TME may stimulate the conversion of ILC2s into other ILC subsets within the tumor tissues, suggesting that the environment that they are exposed to can dictate their pro- and/or anti-tumoral roles. In the nasal polyps of cystic fibrosis patients, ILC2s are reported to be capable of differentiation into IL-17 producing cells when stimulated with IL-1 $\beta$, IL-23, and TGF- $\beta$, the concomitant downregulation of GATA-3 and increased expression of ROR $\gamma \mathrm{t}$ were also observed (108). Therefore, ILC2s may be detrimental in the pathogenesis of IL-17-associated diseases, including some 
types of cancer. Efforts to understand the role of bona fide and/or plastic ILC2s in tumors represents the next challenging step. In this endeavor, the use of mouse models will be crucial, for example, the use of genetically engineered ILC2-depleted mice would allow dissection of the real contribution of this cell type to tumor development and/or progression (109). However, ILC2 characterization at any given time point in tumor-bearing mice will always be difficult due to their inherent plasticity. Moreover, the contribution of nILC2s and iILC2s remains to be elucidated in the tumor setting. Given their different abilities to respond to cytokines and, therefore, their potential distinct pro- and/or antitumor roles, further investigation should consider both subsets separately. The use of reporter mice, such as the $\mathrm{Il1} 13^{\mathrm{GFP}}$ or other type- 2 cytokine reporter animals, represent helpful tools to track ILC2s, independently of their transcriptional profiles that can be shaped by the interaction with tumor cells and/or by the TME. The use of humanized mice (BRGST HIS mice) to establish patient-derived xenograft (PDX) models would provide unique environments for interrogation of the function of the innate immune system, in particular, the contribution of ILC2s to cancer development and progression (110). Collectively, these strategies are expected to accelerate our knowledge of ILC2 biology, and provide new insight into potential therapeutic targets. One approach may be to target Th2-associated cytokines and ILC2secreted molecules using neutralizing antibodies. This is the same technique employed by some NK cell-based immunotherapies, for example the use of a transforming growth factor beta (TGF$\beta$ ) antibody to block TGF- $\beta$ signaling, restores NK anti-tumor activity and synergy with $\alpha-\mathrm{PD}-1$ can be achieved (111). These anti-cytokine-based immunotherapies may also be effective for altering ILC2 function. However, they are a challenging and non-specific target due to the diversity of cell types producing

\section{REFERENCES}

1. Spits H, Artis D, Colonna M, Diefenbach A, Di Santo JP, Eberl G, et al. Innate lymphoid cells-a proposal for uniform nomenclature. Nat Rev Immunol. (2013) 13:145-9. doi: 10.1038/nri3365

2. Moro K, Yamada T, Tanabe M, Takeuchi T, Ikawa T, Kawamoto $\mathrm{H}$, et al. Innate production of $\mathrm{T}(\mathrm{H}) 2$ cytokines by adipose tissueassociated c-Kit(+)Sca-1(+) lymphoid cells. Nature. (2010) 463:540-4. doi: $10.1038 /$ nature08636

3. Neill DR, Wong SH, Bellosi A, Flynn RJ, Daly M, Langford TK, et al. Nuocytes represent a new innate effector leukocyte that mediates type-2 immunity. Nature. (2010) 464:1367-70. doi: 10.1038/nature08900

4. Price AE, Liang HE, Sullivan BM, Reinhardt RL, Eisley CJ, Erle DJ, et al. Systemically dispersed innate IL-13-expressing cells in type 2 immunity. Proc Natl Acad Sci USA. (2010) 107:11489-94. doi: 10.1073/pnas.1003988107

5. Kim BS, Siracusa MC, Saenz SA, Noti M, Monticelli LA, Sonnenberg GF, et al. TSLP elicits IL-33-independent innate lymphoid cell responses to promote skin inflammation. Sci Transl Med. (2013) 5:170ra116. doi: 10.1126/scitranslmed.3005374

6. Sonnenberg GF, Artis D. Innate lymphoid cells in the initiation, regulation and resolution of inflammation. Nat Med. (2015) 21:698-708. doi: $10.1038 / \mathrm{nm} .3892$

7. Yazdani R, Sharifi M, Shirvan AS, Azizi G, Ganjalikhani-Hakemi M. Characteristics of innate lymphoid cells (ILCs) and their role in immunological disorders (an update). Cell Immunol. (2015) 298:66-76. doi: 10.1016/j.cellimm.2015.09.006 them and their multiple roles in different physiological and pathophysiological processes. Another attractive strategy for targeting ILC2s may involve the disruption of transcription factor signatures, that are emerging as indispensable in ILC2 biology, or the manipulation of their metabolic programs. Lastly, remarkable success has been recently achieved in the clinics by immunotherapy based on immune checkpoint blockade, including agents targeting CTLA4, PD-1, or PD-L1. While the pattern of CTLA4 and PD-1 expression in various subsets of $\mathrm{CD}^{+} \mathrm{T}$ and $\mathrm{CD} 8^{+} \mathrm{T}$ cells are well-understood, little is known on the expression of immune checkpoints in ILCs. Of note, PD1 has been reported as an intrinsic negative regulator of the functions of the ILC2 subset in mice, raising the possibility that current treatments targeting PD-1 might significantly impact on ILC functions (112). Ultimately with constant new discoveries in the ILC2 field in health and disease, immunotherapies focusing on the functional targeting of ILC2 are fast approaching clinical realization.

\section{AUTHOR CONTRIBUTIONS}

GE, MF, and GV wrote the manuscript. ST and CJ wrote and critically revised the manuscript. All authors provided approval for publication of the manuscript.

\section{FUNDING}

This work was supported by grants from the Swiss National Science Foundation (PZ00P3_161459 and PR00P3_179727, to CJ), the Swiss Cancer League (KFS-3710-08-2015-R, to CJ), a ProFemmes UNIL fellowship (to ST), and by the Ludwig Institute for Cancer Research.
8. Vivier E, Artis D, Colonna M, Diefenbach A, Di Santo JP, Eberl G, et al. Innate lymphoid cells: 10 years on. Cell. (2018) 174:1054-66. doi: 10.1016/j.cell.2018.07.017

9. Bernink JH, Peters CP, Munneke M, Te Velde AA, Meijer SL, Weijer K, et al. Human type 1 innate lymphoid cells accumulate in inflamed mucosal tissues. Nat Immunol. (2013) 14:221-9. doi: 10.1038/ni.2534

10. Luci C, Reynders A, Ivanov Ii, Cognet C, Chiche L, Chasson L, et al. Influence of the transcription factor RORgammat on the development of NKp46+ cell populations in gut and skin. Nat Immunol. (2009) 10:75-82. doi: $10.1038 /$ ni.1681

11. Hochdorfer T, Winkler C, Pardali K, Mjosberg J. Expression of cKit discriminates between two functionally distinct subsets of human type 2 innate lymphoid cells. Eur J Immunol. (2019) 49:884-93. doi: 10.1002/eji.201848006

12. Nagasawa M, Heesters BA, Kradolfer CMA, Krabbendam L, MartinezGonzalez I, De Bruijn MJW, et al. Correction: KLRG1 and NKp46 discriminate subpopulations of human CD117(+)CRTH2(-) ILCs biased toward ILC2 or ILC3. J Exp Med. (2019) 216:2221-2. doi: 10.1084/jem.2019049007302019c

13. Voehringer D, Koschella M, Pircher H. Lack of proliferative capacity of human effector and memory $\mathrm{T}$ cells expressing killer cell lectinlike receptor G1 (KLRG1). Blood. (2002) 100:3698-702. doi: 10.1182/blood-2002-02-0657

14. Ito M, Maruyama T, Saito N, Koganei S, Yamamoto K, Matsumoto N. Killer cell lectin-like receptor G1 binds three members of the classical cadherin family to inhibit NK cell cytotoxicity. J Exp Med. (2006) 203:289-95. doi: 10.1084/jem.20051986 
15. Huang Y, Guo L, Qiu J, Chen X, Hu-Li J, Siebenlist U, et al. IL-25-responsive, lineage-negative KLRG1(hi) cells are multipotential 'inflammatory' type 2 innate lymphoid cells. Nat Immunol. (2015) 16:161-9. doi: 10.1038/ni.3078

16. Cardoso V, Chesne J, Ribeiro H, Garcia-Cassani B, Carvalho T, Bouchery T, et al. Neuronal regulation of type 2 innate lymphoid cells via neuromedin $\mathrm{U}$. Nature. (2017) 549:277-81. doi: 10.1038/nature23469

17. Klose CSN, Mahlakoiv T, Moeller JB, Rankin LC, Flamar AL, Kabata H, et al. The neuropeptide neuromedin $\mathrm{U}$ stimulates innate lymphoid cells and type 2 inflammation. Nature. (2017) 549:282-6. doi: 10.1038/nature23676

18. Wallrapp A, Riesenfeld SJ, Burkett PR, Abdulnour RE, Nyman J, Dionne $\mathrm{D}$, et al. The neuropeptide NMU amplifies ILC2-driven allergic lung inflammation. Nature. (2017) 549:351-6. doi: 10.1038/nature24029

19. Maric J, Ravindran A, Mazzurana L, Bjorklund AK, Van Acker A, Rao A, et al. Prostaglandin E2 suppresses human group 2 innate lymphoid cell function. $J$ Allergy Clin Immunol. (2018) 141:1761-73.e6. doi: 10.1016/j.jaci.2017.09.050

20. Xu H, Ding J, Porter CBM, Wallrapp A, Tabaka M, Ma S, et al. Transcriptional atlas of intestinal immune cells reveals that neuropeptide alpha-CGRP modulates group 2 innate lymphoid cell responses. Immunity. (2019) 51:696-708.e9. doi: 10.1016/j.immuni.2019.09.004

21. Sui P, Wiesner DL, Xu J, Zhang Y, Lee J, Van Dyken S, et al. Pulmonary neuroendocrine cells amplify allergic asthma responses. Science. (2018) 360:eaan8546. doi: 10.1126/science.aan8546

22. Nussbaum JC, Van Dyken SJ, Von Moltke J, Cheng LE, Mohapatra A, Molofsky $\mathrm{AB}$, et al. Type 2 innate lymphoid cells control eosinophil homeostasis. Nature. (2013) 502:245-8. doi: 10.1038/nature12526

23. Nagashima H, Mahlakoiv T, Shih HY, Davis FP, Meylan F, Huang Y, et al. Neuropeptide CGRP limits group 2 innate lymphoid cell responses and constrains type 2 inflammation. Immunity. (2019) 51:682-95.e6. doi: 10.1016/j.immuni.2019.06.009

24. Wallrapp A, Burkett PR, Riesenfeld SJ, Kim SJ, Christian E, Abdulnour RE, et al. Calcitonin gene-related peptide negatively regulates alarmin-driven type 2 innate lymphoid cell responses. Immunity. (2019) 51:709-23.e6. doi: 10.1016/j.immuni.2019.09.005

25. Moriyama S, Brestoff JR, Flamar AL, Moeller JB, Klose CSN, Rankin LC, et al. beta2-adrenergic receptor-mediated negative regulation of group 2 innate lymphoid cell responses. Science. (2018) 359:1056-61. doi: 10.1126/science.aan4829

26. Monticelli LA, Sonnenberg GF, Abt MC, Alenghat T, Ziegler CG, Doering TA, et al. Innate lymphoid cells promote lung-tissue homeostasis after infection with influenza virus. Nat Immunol. (2011) 12:1045-54. doi: $10.1038 /$ ni.2131

27. Maric J, Ravindran A, Mazzurana L, Van Acker A, Rao A, Kokkinou E, et al. Cytokine-induced endogenous production of prostaglandin D2 is essential for human group 2 innate lymphoid cell activation. J Allergy Clin Immunol. (2019) 143:2202-14.e25. doi: 10.1016/j.jaci.2018.10.069

28. Moffatt MF, Gut IG, Demenais F, Strachan DP, Bouzigon E, Heath $\mathrm{S}$, et al. A large-scale, consortium-based genomewide association study of asthma. N Engl J Med. (2010) 363:1211-21. doi: 10.1056/NEJMoa 0906312

29. Jeffery HC, Mcdowell P, Lutz P, Wawman RE, Roberts S, Bagnall C, et al. Human intrahepatic ILC2 are IL-13positive amphiregulinpositive and their frequency correlates with model of end stage liver disease score. PLOS ONE. (2017) 12:e0188649. doi: 10.1371/journal.pone.0188649

30. Zhang J, Han S, Zhang B, Zhang Y. Cancer immunology and cancer immunodiagnosis. J Immunol Res. (2014) 2014:725691. doi: 10.1155/2014/725691

31. Trabanelli S, Chevalier MF, Martinez-Usatorre A, Gomez-Cadena A, Salome B, Lecciso M, et al. Tumour-derived PGD2 and NKp30-B7H6 engagement drives an immunosuppressive ILC2-MDSC axis. Nat Commun. (2017) 8:593. doi: 10.1038/s41467-017-00678-2

32. Salimi M, Wang R, Yao X, Li X, Wang X, Hu Y, et al. Activated innate lymphoid cell populations accumulate in human tumour tissues. BMC Cancer. (2018) 18:341. doi: 10.1186/s12885-018-4262-4

33. Jovanovic IP, Pejnovic NN, Radosavljevic GD, Pantic JM, Milovanovic $\mathrm{MZ}$, Arsenijevic NN, et al. Interleukin-33/ST2 axis promotes breast cancer growth and metastases by facilitating intratumoral accumulation of immunosuppressive and innate lymphoid cells. Int J Cancer. (2014) 134:1669-82. doi: 10.1002/ijc.28481
34. Suzuki A, Leland P, Joshi BH, Puri RK. Targeting of IL-4 and IL-13 receptors for cancer therapy. Cytokine. (2015) 75:79-88. doi: $10.1016 /$ j.cyto.2015.05.026

35. Ostrand-Rosenberg S, Sinha P. Myeloid-derived suppressor cells: linking inflammation and cancer. J Immunol. (2009) 182:4499-506. doi: 10.4049/jimmunol.0802740

36. Zaiss DM, Van Loosdregt J, Gorlani A, Bekker CP, Grone A, Sibilia $\mathrm{M}$, et al. Amphiregulin enhances regulatory $\mathrm{T}$ cell-suppressive function via the epidermal growth factor receptor. Immunity. (2013) 38:275-84. doi: 10.1016/j.immuni.2012.09.023

37. Simson L, Ellyard JI, Dent LA, Matthaei KI, Rothenberg ME, Foster PS, et al. Regulation of carcinogenesis by IL-5 and CCL11: a potential role for eosinophils in tumor immune surveillance. J Immunol. (2007) 178:4222-9. doi: 10.4049/jimmunol.178.7.4222

38. Krok-Schoen JL, Fisher JL, Stephens JA, Mims A, Ayyappan S, Woyach JA, et al. Incidence and survival of hematological cancers among adults ages $>/=75$ years. Cancer Med. (2018) 7:3425-33. doi: 10.1002/cam4.1461

39. Hazenberg MD, Spits H. Human innate lymphoid cells. Blood. (2014) 124:700-9. doi: 10.1182/blood-2013-11-427781

40. Trabanelli S, Curti A, Lecciso M, Salome B, Riether C, Ochsenbein A, et al. $\mathrm{CD} 127+$ innate lymphoid cells are dysregulated in treatment naive acute myeloid leukemia patients at diagnosis. Haematologica. (2015) 100:e257-60. doi: 10.3324/haematol.2014.119602

41. Kim J, Kim W, Moon UJ, Kim HJ, Choi HJ, Sin JI, et al. Intratumorally establishing type 2 innate lymphoid cells blocks tumor growth. J Immunol. (2016) 196:2410-23. doi: 10.4049/jimmunol.1501730

42. Siegel RL, Miller KD, Jemal A. Cancer statistics, 2017. CA Cancer J Clin. (2017) 67:7-30. doi: 10.3322/caac.21387

43. Nevedomskaya E, Baumgart SJ, Haendler B. Recent advances in prostate cancer treatment and drug discovery. Int J Mol Sci. (2018) 19:E1359. doi: 10.3390/ijms19051359

44. Corn PG. The tumor microenvironment in prostate cancer: elucidating molecular pathways for therapy development. Cancer Manag Res. (2012) 4:183-93. doi: 10.2147/CMAR.S32839

45. Wu CP, Hsiao SH, Murakami M, Lu MJ, Li YQ, Hsieh CH, et al. Tyrphostin RG14620 selectively reverses ABCG2-mediated multidrug resistance in cancer cell lines. Cancer Lett. (2017) 409:56-65. doi: 10.1016/j.canlet.2017.08.035

46. Greenberg NM, Demayo F, Finegold MJ, Medina D, Tilley WD, Aspinall JO, et al. Prostate cancer in a transgenic mouse. Proc Natl Acad Sci USA. (1995) 92:3439-43. doi: 10.1073/pnas.92.8.3439

47. Saranchova I, Han J, Zaman R, Arora H, Huang H, Fenninger F, et al. Type 2 innate lymphocytes actuate immunity against tumours and limit cancer metastasis. Sci Rep. (2018) 8:2924. doi: 10.1038/s41598-018-20608-6

48. Cumberbatch MGK, Jubber I, Black PC, Esperto F, Figueroa JD, Kamat $\mathrm{AM}$, et al. Epidemiology of bladder cancer: a systematic review and contemporary update of risk factors in 2018. Eur Urol. (2018) 74:784-95. doi: 10.1016/j.eururo.2018.09.001

49. Babjuk M, Bohle A, Burger M, Capoun O, Cohen D, Comperat EM, et al. EAU guidelines on non-muscle-invasive urothelial carcinoma of the bladder: update 2016. Eur Urol. (2017) 71:447-61. doi: 10.1016/j.eururo.2016.05.041

50. Matulay JT, Kamat AM. Advances in risk stratification of bladder cancer to guide personalized medicine. F1000Res. (2018). 7. doi: 10.12688/f1000research.14903.1

51. Kamat AM, Sylvester RJ, Bohle A, Palou J, Lamm DL, Brausi M, et al. Definitions, end points, and clinical trial designs for non-muscle-invasive bladder cancer: recommendations from the international bladder cancer group. J Clin Oncol. (2016) 34:1935-44. doi: 10.1200/JCO.2015.64.4070

52. Chevalier MF, Trabanelli S, Racle J, Salome B, Cesson V, Gharbi D, et al. ILC2-modulated T cell-to-MDSC balance is associated with bladder cancer recurrence. J Clin Invest. (2017) 127:2916-29. doi: 10.1172/JCI89717

53. Benard F, Barkun AN, Martel M, Von Renteln D. Systematic review of colorectal cancer screening guidelines for average-risk adults: Summarizing the current global recommendations. World J Gastroenterol. (2018) 24:12438. doi: 10.3748/wjg.v24.i1.124

54. Shaukat A, Mongin SJ, Geisser MS, Lederle FA, Bond JH, Mandel JS, et al. Long-term mortality after screening for colorectal cancer. $N$ Engl J Med. (2013) 369:1106-14. doi: 10.1056/NEJMoa1300720 
55. Thanikachalam K, Khan G. Colorectal cancer and nutrition. Nutrients. (2019) 11:164. doi: 10.3390/nu11010164

56. Zhang L, Zhao Y, Dai Y, Cheng JN, Gong Z, Feng Y, et al. Immune landscape of colorectal cancer tumor microenvironment from different primary tumor location. Front Immunol. (2018) 9:1578. doi: 10.3389/fimmu.2018.01578

57. Cho HS, Reboldi A, Hall JA, Berg LJ. The Tec kinase ITK is essential for ILC2 survival and epithelial integrity in the intestine. Nat Commun. (2019) 10:784. doi: 10.1038/s41467-019-08699-9

58. Simoni Y, Fehlings M, Kloverpris HN, Mcgovern N, Koo SL, Loh CY, et al. Human innate lymphoid cell subsets possess tissue-type based heterogeneity in phenotype and frequency. Immunity. (2017) 46:148-61. doi: 10.1016/j.immuni.2016.11.005

59. Maywald RL, Doerner SK, Pastorelli L, De Salvo C, Benton SM, Dawson EP, et al. IL-33 activates tumor stroma to promote intestinal polyposis. Proc Natl Acad Sci USA. (2015) 112:E2487-96. doi: 10.1073/pnas.1422445112

60. Akimoto M, Maruyama R, Takamaru H, Ochiya T, Takenaga K. Soluble IL-33 receptor sST2 inhibits colorectal cancer malignant growth by modifying the tumour microenvironment. Nat Commun. (2016) 7:13589. doi: $10.1038 /$ ncomms 13589

61. Mertz KD, Mager LF, Wasmer MH, Thiesler T, Koelzer VH, Ruzzante G, et al. The IL-33/ST2 pathway contributes to intestinal tumorigenesis in humans and mice. Oncoimmunology. (2016) 5:e1062966. doi: 10.1080/2162402X.2015.1062966

62. Li Y, Shi J, Qi S, Zhang J, Peng D, Chen Z, et al. IL-33 facilitates proliferation of colorectal cancer dependent on $\mathrm{COX}_{2}$ /PGE2. J Exp Clin Cancer Res. (2018) 37:196. doi: 10.1186/s13046-018-0839-7

63. Shen JX, Liu J, Zhang GJ. Interleukin-33 in Malignancies: Friends or Foes? Front Immunol. (2018) 9:3051. doi: 10.3389/fimmu.2018.03051

64. Busser B, Sancey L, Brambilla E, Coll JL, Hurbin A. The multiple roles of amphiregulin in human cancer. Biochim Biophys Acta. (2011) 1816:119-31. doi: 10.1016/j.bbcan.2011.05.003

65. Chayangsu C, Khunsri S, Sriuranpong V, Tanasanvimon S. The correlations between serum amphiregulin and other clinicopathological factors in colorectal cancer. J Gastrointest Oncol. (2017) 8:980-4. doi: 10.21037/jgo.2017.08.15

66. Kinugasa T, Akagi Y, Ochi T, Tanaka N, Kawahara A, Ishibashi Y, et al. Increased claudin-1 protein expression in hepatic metastatic lesions of colorectal cancer. Anticancer Res. (2012) 32:2309-14.

67. Grundker C, Lasche M, Hellinger JW, Emons G. Mechanisms of metastasis and cell mobility - the role of metabolism. Geburtshilfe Frauenheilkd. (2019) 79:184-8. doi: 10.1055/a-0805-9113

68. Zaiss DMW, Gause WC, Osborne LC, Artis D. Emerging functions of amphiregulin in orchestrating immunity, inflammation, and tissue repair. Immunity. (2015) 42:216-26. doi: 10.1016/j.immuni.2015.01.020

69. Thrift AP, El-Serag HB. Burden of gastric cancer. Clin Gastroenterol Hepatol. (2019). doi: 10.1016/j.cgh.2019.07.045. [Epub ahead of print]

70. Song Z, Wu Y, Yang J, Yang D, Fang X. Progress in the treatment of advanced gastric cancer. Tumour Biol. (2017) 39, 1-7. doi: 10.1177/10104283177 14626

71. De Guillebon E, Roussille P, Frouin E, Tougeron D. Anti program death1/anti program death-ligand 1 in digestive cancers. World J Gastrointest Oncol. (2015) 7:95-101. doi: 10.4251/wjgo.v7.i8.95

72. Yusefi AR, Bagheri Lankarani K, Bastani P, Radinmanesh M, Kavosi Z. Risk factors for gastric cancer: a systematic review. Asian Pac J Cancer Prev. (2018) 19:591-603. doi: 10.22034/APJCP.2018.19.3.591

73. Den Hoed CM, Kuipers EJ. Gastric cancer: how can we reduce the incidence of this disease? Curr Gastroenterol Rep. (2016) 18:34. doi: 10.1007/s11894-016-0506-0

74. Li R, Jiang XX, Zhang LF, Liu XM, Hu TZ, Xia XJ, et al. Group 2 innate lymphoid cells are involved in skewed type 2 immunity of gastric diseases induced by Helicobacter pylori infection. Mediators Inflamm. (2017) 2017:4927964. doi: 10.1155/2017/4927964

75. Monticelli LA, Buck MD, Flamar AL, Saenz SA, Tait Wojno ED, Yudanin NA, et al. Arginase 1 is an innate lymphoid-cell-intrinsic metabolic checkpoint controlling type 2 inflammation. Nat Immunol. (2016) 17:65665. doi: 10.1038/ni.3421

76. Suwanpradid J, Shih M, Pontius L, Yang B, Birukova A, Guttman-Yassky E, et al. Arginasel deficiency in monocytes/macrophages upregulates inducible nitric oxide synthase to promote cutaneous contact hypersensitivity. $J$ Immunol. (2017) 199:1827-34. doi: 10.4049/jimmunol.1700739

77. Bie Q, Zhang P, Su Z, Zheng D, Ying X, Wu Y, et al. Polarization of ILC2s in peripheral blood might contribute to immunosuppressive microenvironment in patients with gastric cancer. J Immunol Res. (2014) 2014:923135. doi: 10.1155/2014/923135

78. Besnard AG, Guabiraba R, Niedbala W, Palomo J, Reverchon F, Shaw $\mathrm{TN}$, et al. IL-33-mediated protection against experimental cerebral malaria is linked to induction of type 2 innate lymphoid cells, M2 macrophages and regulatory T cells. PLoS Pathog. (2015) 11:e1004607. doi: 10.1371/journal.ppat.1004607

79. Saleh R, Elkord E. Acquired resistance to cancer immunotherapy: Role of tumor-mediated immunosuppression. Semin Cancer Biol. (2019). doi: 10.1016/j.semcancer.2019.07.017. [Epub ahead of print].

80. Eissmann MF, Dijkstra C, Jarnicki A, Phesse T, Brunnberg J, Poh $\mathrm{AR}$, et al. IL-33-mediated mast cell activation promotes gastric cancer through macrophage mobilization. Nat Comm. 10:2735 (2019) doi: $10.1038 /$ s41467-019-10676

81. Anastasiadi Z, Lianos GD, Ignatiadou E, Harissis HV, Mitsis M. Breast cancer in young women: an overview. Updates Surg. (2017) 69:313-7. doi: 10.1007/s13304-017-0424-1

82. Merino Bonilla JA, Torres Tabanera M, Ros Mendoza LH. Breast cancer in the 21st century: from early detection to new therapies. Radiologia. (2017) 59:368-79. doi: 10.1016/j.rxeng.2017.08.001

83. Barkal AA, Brewer RE, Markovic M, Kowarsky M, Barkal SA, Zaro BW, et al. CD24 signalling through macrophage Siglec-10 is a target for cancer immunotherapy. Nature. (2019). doi: 10.1038/s41586-019-1456-0

84. Stovgaard ES, Dyhl-Polk A, Roslind A, Balslev E, Nielsen D. PDL1 expression in breast cancer: expression in subtypes and prognostic significance: a systematic review. Breast Cancer Res Treat. (2019) 174:571-84. doi: 10.1007/s10549-019-05130-1

85. Perou CM, Sorlie T, Eisen MB, Van De Rijn M, Jeffrey SS, Rees CA, et al. Molecular portraits of human breast tumours. Nature. (2000) 406:747-52. doi: $10.1038 / 35021093$

86. Disis ML, Stanton SE. Immunotherapy in breast cancer: an introduction. Breast. (2018) 37:196-9. doi: 10.1016/j.breast.2017.01.013

87. Schmucker H, Blanding WM, Mook JM, Wade JF, Park JP, Kwist K, et al. Amphiregulin regulates proliferation and migration of HER2-positive breast cancer cells. Cell Oncol. (2018) 41:159-68. doi: 10.1007/s13402-017-0363-3

88. Davis LE, Shalin SC, Tackett AJ. Current state of melanoma diagnosis and treatment. Cancer Biol Ther. (2019) 20:1366-79. doi: 10.1080/15384047.2019.1640032

89. Luke JJ, Flaherty KT, Ribas A, Long GV. Targeted agents and immunotherapies: optimizing outcomes in melanoma. Nat Rev Clin Oncol. (2017) 14:463-82. doi: 10.1038/nrclinonc.2017.43

90. Nowicki TS, Berent-Maoz B, Cheung-Lau G, Huang RR, Wang X, Tsoi J, et al. A pilot trial of the combination of transgenic NY-ESO-1-reactive adoptive cellular therapy with dendritic cell vaccination with or without ipilimumab. Clin Cancer Res. (2019) 25:2096-108. doi: 10.1158/1078-0432.CCR-18-3496

91. Ebiomedicine The tumor microenvironment: a druggable target for metastatic disease? EBioMedicine. (2018) 31:1-2. doi: 10.1016/j.ebiom.2018.05.005

92. Long A, Dominguez D, Qin L, Chen S, Fan J, Zhang M, et al. Type 2 innate lymphoid cells impede IL-33-mediated tumor suppression. J Immunol. (2018) 201:3456-64. doi: 10.4049/jimmunol.1800173

93. Ikutani M, Yanagibashi T, Ogasawara M, Tsuneyama K, Yamamoto S, Hattori $\mathrm{Y}$, et al. Identification of innate IL-5-producing cells and their role in lung eosinophil regulation and antitumor immunity. J Immunol. (2012) 188:70313. doi: 10.4049/jimmunol.1101270

94. Zhang Y, Yang Q, Wang S. MicroRNAs: a new key in lung cancer. Cancer Chemother Pharmacol. (2014) 74:1105-11. doi: 10.1007/s00280-014-2559-9

95. Ray G, Henson DE, Schwartz AM. Cigarette smoking as a cause of cancers other than lung cancer: an exploratory study using the surveillance, epidemiology, and end results program. Chest. (2010) 138:4919. doi: 10.1378/chest.09-1909

96. Hirsch FR, Scagliotti GV, Mulshine JL, Kwon R, Curran WJJr, Wu YL, et al. Lung cancer: current therapies and new targeted treatments. Lancet. (2017) 389:299-311. doi: 10.1016/S0140-6736(16)30958-8 
97. Lemjabbar-Alaoui H, Hassan OU, Yang YW, Buchanan P. Lung cancer: Biology and treatment options. Biochim Biophys Acta. (2015) 1856:189-210. doi: 10.1016/j.bbcan.2015.08.002

98. Reinmuth N, Reck M. Immunotherapy for lung cancer. Oncol Res Treat. (2016) 39:360-8. doi: 10.1159/000446726

99. Yang S, Zhang Z, Wang Q. Emerging therapies for small cell lung cancer. J Hematol Oncol. (2019) 12:47. doi: 10.1186/s13045-01 9-0736-3

100. Dammert MA, Bragelmann J, Olsen RR, Bohm S, Monhasery N, Whitney $\mathrm{CP}$, et al. MYC paralog-dependent apoptotic priming orchestrates a spectrum of vulnerabilities in small cell lung cancer. Nat Commun. (2019) 10:3485. doi: 10.1038/s41467-019-11371-x

101. Regzedmaa O, Zhang H, Liu H, Chen J. Immune checkpoint inhibitors for small cell lung cancer: opportunities and challenges. Onco Targets Ther. (2019) 12:4605-20. doi: 10.2147/OTT.S204577

102. Halim TY, Steer CA, Matha L, Gold MJ, Martinez-Gonzalez I, Mcnagny $\mathrm{KM}$, et al. Group 2 innate lymphoid cells are critical for the initiation of adaptive $\mathrm{T}$ helper 2 cell-mediated allergic lung inflammation. Immunity. (2014) 40:425-35. doi: 10.1016/j.immuni.2014.01.011

103. Wu Y, Yan Y, Su Z, Bie Q, Chen X, Barnie PA, et al. Enhanced circulating ILC2s and MDSCs may contribute to ensure maintenance of Th2 predominant in patients with lung cancer. Mol Med Rep. (2017) 15:4374-81. doi: $10.3892 / \mathrm{mmr} .2017 .6537$

104. Carrega P, Loiacono F, Di Carlo E, Scaramuccia A, Mora M, Conte $\mathrm{R}$, et al. NCR(+)ILC3 concentrate in human lung cancer and associate with intratumoral lymphoid structures. Nat Commun. (2015) 6:8280. doi: $10.1038 /$ ncomms 9280

105. Wu S, Zhu W, Thompson P, Hannun YA. Evaluating intrinsic and non-intrinsic cancer risk factors. Nat Commun. (2018) 9:3490. doi: 10.1038/s41467-018-05467-z

106. Gonzalez H, Hagerling C, Werb Z. Roles of the immune system in cancer: from tumor initiation to metastatic progression. Genes Dev. (2018) 32:126784. doi: $10.1101 /$ gad.314617.118
107. Trabanelli S, Chevalier MF, Derre L, Jandus C. The pro- and anti-tumor role of ILC2s. Semin Immunol. (2019) 41:101276. doi: 10.1016/j.smim.2019.04.004

108. Golebski K, Ros XR, Nagasawa M, Van Tol S, Heesters BA, Aglmous H, et al. IL-1beta, IL-23, and TGF-beta drive plasticity of human ILC2s towards IL-17-producing ILCs in nasal inflammation. Nat Commun. (2019) 10:2162. doi: 10.1038/s41467-019-09883-7

109. Oliphant CJ, Hwang YY, Walker JA, Salimi M, Wong SH, Brewer JM, et al. MHCII-mediated dialog between group 2 innate lymphoid cells and CD4(+) $\mathrm{T}$ cells potentiates type 2 immunity and promotes parasitic helminth expulsion. Immunity. (2014) 41:283-95. doi: 10.1016/j.immuni.2014. 06.016

110. Li Y, Masse-Ranson G, Garcia Z, Bruel T, Kok A, Strick-Marchand $\mathrm{H}$, et al. A human immune system mouse model with robust lymph node development. Nat Methods. (2018) 15:623-30. doi: 10.1038/s41592-01 8-0071-6

111. Akhurst RJ, Hata A. Targeting the TGFbeta signalling pathway in disease. Nat Rev Drug Discov. (2012) 11:790-811. doi: 10.1038/nrd3810

112. Taylor S, Huang Y, Mallett G, Stathopoulou C, Felizardo TC, Sun MA, et al. PD-1 regulates KLRG1(+) group 2 innate lymphoid cells. J Exp Med. (2017) 214:1663-78. doi: 10.1084/jem.20161653

Conflict of Interest: The authors declare that the research was conducted in the absence of any commercial or financial relationships that could be construed as a potential conflict of interest.

Copyright (C) 2019 Ercolano, Falquet, Vanoni, Trabanelli and Jandus. This is an open-access article distributed under the terms of the Creative Commons Attribution License (CC BY). The use, distribution or reproduction in other forums is permitted, provided the original author(s) and the copyright owner(s) are credited and that the original publication in this journal is cited, in accordance with accepted academic practice. No use, distribution or reproduction is permitted which does not comply with these terms. 